$\square$

As a result of alkaline neutralization of oils, a significant amount of soapstock is formed, the utilization of which creates an environmental and economic problem. The production of fatty acids from soapstock using sulfuric acid decomposition is investigated in this work.

The peculiarity of the work is the determination of regression dependences of the yield and neutralization number of fatty acids on the soapstock processing conditions: temperature and duration.

Soapstock obtained after neutralization of sunflower oil was used as raw material. Soapstock indicators: mass fraction of moisture - $15.4 \%$, total fat $-71.9 \%$, fatty acids $-64.5 \%$, neutral fat $-7.4 \%$.

Rational conditions of soapstock processing are determined: temperature (90-95) ${ }^{\circ} \mathrm{C}$, duration $40 \mathrm{~min}$. Under these conditions, the fatty acid yield is $79.0 \%$, the neutralization number is $180.0 \mathrm{mg} \mathrm{KOH} / \mathrm{g}$. Quality indicators of the obtained fatty acids: mass fraction of moisture and volatile substances $1.8 \%$, mass fraction of total fat $97.0 \%$, cleavage depth $-64.5 \%$ of oleic acid, the presence of mineral acids - no. Fatty acids correspond to fatty acids of the first grade according to DSTU 4860 (CAS 61788-66-7).

An increase in the temperature and duration of soapstock contact with sulfuric acid increases the yield and neutralization number of fatty acids. This is due to a decrease in the viscosity of the reaction medium, an increase in the depth of cleavage of soapstock soaps with sulfuric acid, an increase in the intensity and duration of mass transfer.

The developed rational conditions allow obtaining fatty acids from soapstock, which correspond in composition to fatty acids from refined deodorized sunflower oil.

The results allow solving a number of economic and environmental problems associated with soapstock utilization and can be implemented in oil refineries and fatty acid production

Keywords: oil and fat industry waste, alkaline neutralization, soapstock, fatty acids, sunflower oil
UDC 665.1

DOI: $10.15587 / 1729-4061.2021 .236984$

\title{
RATIONAL CONDITIONS OF FATTY ACIDS OBTAINING BY SOAPSTOCK TREATMENT WITH SULFURIC ACID
}

Natalia Sytnik

Corresponding author $\mathrm{PhD*}$

E-mail: ntlsytnik@gmail.com

Ek a terina Kunitsia $\mathrm{PhD}$

Department of Innovative Food and Restaurant Technologies Kharkiv Institute of Trade and Economics of Kyiv National University of Trade and Economics

O. Yarosh lane, 8, Kharkiv, Ukraine, 61045

Viktoria Mazaeva

$\mathrm{PhD*}$

Viktoriia Kalyna

$\mathrm{PhD}$, Associate Professor Department of Technology of Storage and Processing of Agricultural Products Dnipro State Agrarian and Economic University Serhiya Yefremova str., 25, Dnipro, Ukraine, 49027

Andri Chernukha**

Serhii Vazhynskyi

$\mathrm{PhD}$, Associate Professor**

O leksandr Y a sh chenko $\mathrm{PhD}$, Associate Professor Department of Management and Organization in the Field of Civil Protection***

Murat Maliarov $\mathrm{PhD}$

Department of Automatic Security Systems and Information Technologies***

O leg Bogatov

$\mathrm{PhD}$, Associate Professor Department of Metrology and Life Safety Kharkiv National Automobile and Highway University Yaroslava Mudroho str., 25, Kharkiv, Ukraine, 61002

Borys Bolibrukh Doctor of Technical Sciences, Associate Professor Department of Civil Security

Lviv Polytechnic National University

S. Bandery str., 12, Lviv, Ukraine, 79013

*Department of Studies of Technology for Processing Oils and Fats Ukrainian Research Institute of Oils and Fats of the National Academy of Agrarian Sciences of Ukraine

Dziuby ave., 2a, Kharkiv, Ukraine, 61019 **Department of Fire Prevention in Settlements*** $\star * \star$ National University of Civil Defence of Ukraine Chernychevska str., 94, Kharkiv, Ukraine, 61023

Received date 24.02.2021 Accepted date 20.04.2021 Published date 31.08.2021
How to Cite: Sytnik, N., Kunitsia, E., Mazaeva, V., Kalyna, V., Chernukha, A., Vazhynskyi, S., Yashchenko, O., Maliarov, M., Bogatov, O., Bolibrukh, B. (2021). Rational conditions of fatty acids obtaining by soapstock treatment with sulfuric acid. Eastern-European Journal of Enterprise Technologies, 4 (6 (112)), 6-13. doi: https://doi.org/10.15587/1729-4061.2021.236984
1. Introduction

Waste and by-products are generated at different stages of oil and fat refining. Thus, for the oil and fat industry, the issues of rational use of secondary products and the development of new low-waste technologies are relevant [1]. One of the main types of waste in the oil and fat industry is soapstock, which is formed as a result of alkaline neutralization 
of oils and fats. Soapstock creates a problem with its processing, storage and disposal. Soapstocks contain moisture, soap, free fatty acids, neutral triacylglycerols, alkali and other substances. Disposal of soapstocks as household waste causes the problem of environmental pollution (soil, water, air of the waste discharge zone) [2]. Soapstocks contain a significant amount of fat (up to $70 \%$ ). In [3], it was noted that fats are capable of oxidation with the formation of toxic products and the release of large amounts of heat.

But soapstock contains components that can be used in various industries. Soapstocks are used in soap making, in surfactants production and textile auxiliaries, as a defoamer, for feed purposes [4]. Soapstock usually requires additional pre-treatment, concentration, and so on. The economic feasibility of using this type of waste must be calculated in each case. It is advisable to use soapstock to obtain fatty acids. Fatty acids are the valuable raw material for many industries.

One of the priority issues in the world is to preserve natural resources and reduce the negative impact of industrial waste on the environment. To this end, new production technologies, methods of wastewater and air emissions treatment are being developed, new types of equipment are being used [5]. In [6], it is noted that the methods of industrial waste management can be classified into three options: reducing the source of pollution by modifying technology, waste recovery, waste processing to obtain valuable products or neutralization of unwanted components. A particularly important area is the rational use of waste, which cannot be avoided. In addition to improving the environmental situation, this will increase the profitability of production through the sale of not only basic products, but also products derived from waste. Thus, the processing of soapstock to obtain fatty acids is an important area of research that helps to improve the environmental state and provide valuable raw materials for production processes in various areas.

\section{Literature review and problem statement}

Soapstock is a large-tonnage waste of the oil and fat industry. Upon obtaining 1 ton of refined oil, (10-20) \% of the soapstock by weight of oil is formed. The value of soapstock is due to the presence of fatty substances in the form of soaps, high molecular weight carboxylic acids and triacylglycerols. The most important component of soapstock is fatty acids.

In [7], it was shown that fatty acids are used for the production of higher fatty alcohols, esters, amides, which are used in the production of surfactants, detergents and cosmetics. However, the profitability of production, the industry's need for these substances and the quality indicators of fatty acids used as raw materials remain open questions.

The thermodynamic properties of fatty acid esters as fuel components are presented in [8]. The thermophysical properties of biodiesel fuel in the high-temperature gas phase have been studied for thermodynamic calculations of piston engine operating processes. In [9], the production and use of hydrocarbon-enriched fuel from soapstock were investigated. The high quality of the obtained product and the possibility of using it as an alternative to other fuels are noted.

The authors [10] noted that compliance with the standards on the toxicity of modern vehicles and special equipment is a topical issue. Various measures are applied: the effect on the engine working process (mixing and combustion processes) and on exhaust gases (neutralization or purification using special systems). Developments in reducing the negative impact of exhaust emissions on the environment are important. In [11], it was shown that even with the minimum addition of biodiesel fuel in the amount of $(2-5) \%$ to petroleum fuel, the toxicity of diesel engine emissions decreases. Thus, biodiesel is widely used in world practice to improve the ecological state of the environment. But an important aspect in biodiesel production is the issue of raw materials.

In [12], it was noted that the cost of sunflower oil reaches $700 \$ / t$, the cost of soapstock (in terms of fats) $-130 \$ / t$. Therefore, oils and fats are not a cost-effective type of raw materials.

The authors [13] showed that promising raw materials for biodiesel fuel are fatty acids from the waste of alkaline neutralization of oils. In studies, the yield of the obtained fatty acid methyl esters was (52-97) \%. But there are still unresolved issues related to measures aimed at steadily increasing ether yield. The ester yield significantly depends on the concentration and moisture content of fatty acids used as raw materials in biodiesel production. Therefore, it is necessary to develop rational conditions and technology for obtaining high-quality fatty acids, which will ensure a high yield of high-quality fuel.

Modern technologies for obtaining fatty acids from soapstock are decomposition by mineral acid. In [7], it was noted that sulfuric acid is most commonly used. The use of hydrochloric acid for decomposition is irrational because its cost is greater than that of sulfuric acid. The use of nitric acid has shown that $20 \%$ of the acid is spent not for the direct reaction of soap decomposition, but for its reduction to nitrogen oxides. This causes an increased consumption. Nitrate waters containing organic compounds (fatty acids) are also formed. This creates a risk of water accumulation in the soil. Therefore, this method is also not used. Thus, developments aimed at efficient production of fatty acids from soapstock by treatment with sulfuric acid are relevant.

In [14], the research results on the isolation of fatty acids by enzymatic and chemical methods are presented. Enzymatic isolation of fatty acids was performed in the presence of a culture of Yarrowia Lipolytica and glycerol at $28{ }^{\circ} \mathrm{C}$ for 48 hours. The chemical method was to treat the soapstock with sulfuric, hydrochloric or orthophosphoric acid. But the disadvantage of the enzymatic method is the significant duration of the process, which makes it impractical. The variation of the parameters of the fatty acid isolation process by these two methods, as well as the qualitative indicators of the obtained fatty acids, in particular, the neutralization number or their concentration in the obtained product are not considered.

The authors [15] investigated the conditions of cotton soapstock decomposition with sulfuric acid with prior hydrolysis of soapstock. The influence of decomposition temperature and acid solution concentration on fatty acid yield and gossypol yield is considered. But it is not shown how the process parameters affect the quality of fatty acids. In addition, this technology is quite complex and multi-stage.

In [16], the scientific results on biodiesel production, which is fatty acid butyl esters, are shown. Three methods of fatty acids obtaining from soapstock in order to obtain esters are considered: treatment with sulfuric acid; saponification and acid treatment; saponification, washing with sodium chloride solution and acid treatment. The disadvantage of the study is the lack of data on the influence of soapstock 
processing parameters on the yield and quality of fatty acids. This is important because the fatty acids indicators affect not only the quality and yield of esters, but also the profitability of biodiesel production from fatty acids derived from soapstock.

Thus, existing studies have shown that an effective modern method of fatty acids extracting from soapstock is sulfuric acid treatment. The influence of some technological parameters of soapstock processing on the fatty acid yield and the efficiency of biodiesel production from them has been studied. But there are still unresolved issues related to the quality of extracted fatty acids, their composition. These data are important because the quality of fatty acids depends on the quality and economic feasibility of production based on them. The characteristics of fatty acids are influenced by the type and indicators of the oil that was subjected to alkaline neutralization; technological parameters of soapstock processing; type and concentration of acid. High-quality fatty acids will increase the profitability of the enterprise through the sale and processing of acids for various purposes.

Therefore, the unresolved issue in processing alkaline neutralization waste is to determine the influence of soapstock treatment conditions on the yield and quality indicators of fatty acids.

\section{The aim and objectives of the study}

The aim of the study was to determine the dependence of the yield and neutralization number of fatty acids on the treatment conditions of oil alkaline neutralization waste (soapstock) with a sulfuric acid solution: temperature and duration of the process. This will make it possible to obtain high-quality fatty acids in industrial conditions and predict the yield and neutralization number of fatty acids

To achieve the aim, the following objectives were set:

- to determine the quality indicators of the industrial sample of soapstock obtained by sunflower oil neutralization;

- to identify the dependence of the yield and neutralization number of fatty acids on the soapstock treatment conditions with sulfuric acid and to determine the rational parameters of soapstock treatment;

- to study the quality indicators and composition of fatty acids obtained under the established rational conditions.

\section{Materials and methods to study the rational conditions for soapstock processing}

\footnotetext{
4. 1. Examined materials and equipment used in the experiment

The following reagents and materials were used in this study:

- rectified ethyl alcohol, according to DSTU 4221:2003 (CAS 64-17-5);

- distilled water, according to acting normative documentation;

- potassium hydroxide, grade "clean for analysis", according to acting normative documentation;

- sodium hydroxide, grade "clean for analysis", according to acting normative documentation;

- phenolphthalein, according to acting normative documentation;
}

- methyl orange, according to acting normative documentation;

- sulfuric acid, grade "clean for analysis", according to GOST 4204 (CAS 7664-93-9);

- ethyl ether, according to acting normative documentation;

- calcium chloride of the highest grade, according to GOST 450 (CAS 10043-52-4).

4. 2. Procedure for determining the quality indicators of the industrial soapstock sample

Organoleptic characteristics of soapstock are determined by the standard method according to DSTU 5033:2008 (Method for determining color, consistency and odor). International methods for determining organoleptic parameters: color - ISO 15305, consistency - AOCS Method Cc 16-60, odor - AOCS Cg 2-83.

The mass fraction of moisture is determined by the standard method according to DSTU 4603:2006 (ISO 662).

The mass fraction of total fat, mass fraction of fatty acids are determined by standard methods according to DSTU 5033:2008 (ISO 17189, IDF 194).

\section{3. Procedure of soapstock treatment with sulfuric} acid solution

In this work, the treatment of soapstock with sulfuric acid was performed as follows. A portion of the soapstock was placed in a heat-resistant conical flask, water with a temperature of $60{ }^{\circ} \mathrm{C}$ in the amount of $50 \%$ by weight of the soapstock was added. The flask is mounted on an electric stove, a stirrer is placed in the flask. While stirring, a solution of sulfuric acid with a concentration of $40 \%$ was added to the flask. The amount of sulfuric acid was adjusted so that excess sulfuric acid was maintained throughout the treatment process (monitoring was performed using the methyl orange indicator or litmus paper). After adding the sulfuric acid solution, the mass was stirred at a given temperature for a given time. The resulting mass was settled for $4-5$ hours. The obtained upper layer of fatty acids was washed with hot water until complete removal of sulfuric acid, which was controlled by methyl orange. The absence of sulfate ions was checked using the calcium chloride solution with a concentration of $10 \%$.

\section{4. Procedure for determining the quality indicators} and composition of fatty acids

The neutralization number of fatty acids is determined as follows. A portion of fatty acids about $2.0 \mathrm{~g}$ is dissolved in (40-60) $\mathrm{cm}^{3}$ of ethyl alcohol. Then, $0.5 \mathrm{~cm}^{3}$ of phenolphthalein solution is added and titrated with $0.5 \mathrm{~N}$ aqueous or alcoholic solution of potassium hydroxide to a pink color that does not disappear within 30 seconds. The neutralization number $(\mathrm{NN})$ is calculated by the formula:

$$
N N=\frac{V \cdot 28.05 \cdot K}{P},
$$

where $V$ - the amount of $\mathrm{cm}^{3} 0.5 \mathrm{~N}$ potassium hydroxide solution, used for titration; 28.05 - titer of exactly $0.5 \mathrm{~N}$ $\mathrm{KOH}$ solution multiplied by $1000 ; K$ - correction to a titer of $0.5 \mathrm{~N}$ potassium hydroxide solution; $P$ - portion of fatty acids, g.

Organoleptic parameters of fatty acids are determined by standard methods according to DSTU 4860:2007. 
The mass fraction of moisture is determined by the standard method according to DSTU 4603:2006.

The mass fraction of total fat, the depth of cleavage, the presence of mineral acids are determined by standard methods according to DSTU 4860:2007.

The composition of the obtained fatty acids and the fatty acid composition of sunflower oil are determined by the standard method according to DSTU ISO 5508-2001 and DSTU ISO 5509-2002.

\section{5. Planning of experimental research and process- ing results \\ In order to plan our study and process the results obtained, a complete second-order factor experiment was used, the calculations of which were performed in the Microsoft Office Excel 2003 (USA) and Stat Soft Statis- tica v6.0 (USA) software packages. The experiments were repeated twice.}

\section{Results of studying the influence of soapstock processing conditions on the efficiency of fatty acid extraction}

5. 1. Determining the quality indicators of the experimental soapstock sample

The quality indicators of the experimental sample of soapstock obtained during the alkaline neutralization of sunflower oil were previously determined. Qualitative indicators of soapstock are presented in Table 1.

Table 1

Qualitative indicators of the experimental soapstock sample

\begin{tabular}{|c|c|}
\hline Indicator & Characteristics \\
\hline \multicolumn{2}{|c|}{ Organoleptic indicators } \\
\hline Color & Brown \\
\hline Consistency at the temperature of $20^{\circ} \mathrm{C}$ & Pasty \\
\hline Odor & $\begin{array}{l}\text { Specific, characteristic } \\
\text { of sunflower oil } \\
\text { soapstock }\end{array}$ \\
\hline \multicolumn{2}{|c|}{ Physico-chemical indicators } \\
\hline $\begin{array}{l}\text { Mass fraction of moisture and volatile } \\
\text { substances, } \%\end{array}$ & 15.4 \\
\hline Mass fraction of total fat, $\%$ & 71.9 \\
\hline Mass fraction of fatty acids, $\%$ & 64.5 \\
\hline Mass fraction of neutral fat, $\%$ & 7.4 \\
\hline
\end{tabular}

Therefore, the experimental soapstock sample has a high value of the mass fraction of total fat and in terms of quality indicators corresponds to DSTU 5033 (CAS 68952-95-4).

5. 2. Identification of the dependence of the yield and neutralization number of fatty acids on the soapstock processing conditions

The influence of soapstock treatment conditions with sulfuric acid on the efficiency of fatty acids obtaining has been determined. The aqueous solution of sulfuric acid with a concentration of $40 \%$ was used in the work. The full factorial experiment was used to conduct research and process the results: the number of factors -2 , the number of experiments -9 , the number of levels $-3[17,18]$.

Factors and variation intervals: $x_{1}$ - temperature of soapstock treatment with sulfuric acid: from 55 to $95^{\circ} \mathrm{C}$; $x_{2}$ - duration of soapstock treatment with sulfuric acid: from 40 to 120 minutes. The response functions were the yield ( $\%$ of the available fatty acid content in soapstock) and neutralization number of fatty acids. Table 2 shows the matrix of experiment planning with the actual values of the factors, as well as the experimentally determined values of the response functions.

As a result of experimental data processing in the Stat Soft Statistica v6.0 (USA) package, mathematical models were obtained that reflect the dependences of response functions on the conditions of soapstock processing. Response functions are marked as follows: $y_{1}$ - yield of fatty acids, $\% ; y_{2}-$ neutralization number of fatty acids, $\mathrm{mg} \mathrm{KOH} / \mathrm{g}$.

In normalized form, the regression dependence of the fatty acid yield on the soapstock processing conditions has the form:

$$
\begin{aligned}
& y_{1}=65.49+10.98 \cdot x_{1}+ \\
& +5.02 \cdot x_{1}^{2}+10.35 \cdot x_{2}+5.02 \cdot x_{2}^{2} .
\end{aligned}
$$

In real variables:

$$
y_{1}=94.21-1.33 \cdot x_{1}+0.01 \cdot x_{1}^{2}-0.24 \cdot x_{2}+0.003 \cdot x_{2}^{2} .
$$

In normalized form, the regression dependence of the neutralization number of fatty acids on the soapstock processing conditions has the form:

$$
\begin{aligned}
& y_{1}=150.09+30.28 \cdot x_{1}- \\
& -2.28 \cdot x_{1}^{2}+14.87 \cdot x_{2}-0.03 \cdot x_{2}^{2} .
\end{aligned}
$$

In real variables:

$$
\begin{aligned}
& y_{1}=-25.45+2.37 \cdot x_{1}- \\
& -0.006 \cdot x_{1}^{2}+0.38 \cdot x_{2}-0.00002 \cdot x_{2}^{2} .
\end{aligned}
$$

No loss of consistency was established (level of significance of regression dependences coefficients $p>0.05$ ). The values of the determination coefficients for the yield and neutralization number of fatty acids were 0.86866 and 0.96439 , respectively (the values are close to unity). Thus, the obtained models adequately describe the response functions.

In dependences (2)-(5): $x_{1}$ - temperature of soapstock processing, ${ }^{\circ} \mathrm{C} ; x_{2}-$ duration of soapstock processing, min. In the equations in normalized form, the values of $x_{1}, x_{2}$ are substituted in coded form (for example, the minimum value of the parameter is denoted by -1 , and the maximum +1$)$. In equations $(3)-(5)$ with real variables, the values of $x_{1}, x_{2}$ in actual dimensions are substituted for calculations.

Table 3 shows the calculated values of the response functions - yield and neutralization number of fatty acids, calculated by equations (3) and (5), respectively. Designations of experiments 1-9 correspond to the planning matrix (Table 2). 
Table 2 Fatty acid yield, \%

Matrix of experiment planning and experimental values of response functions

\begin{tabular}{|c|c|c|c|c|}
\hline $\begin{array}{c}\text { Expe- } \\
\text { riment } \\
\text { number }\end{array}$ & $\begin{array}{c}\text { Tempera- } \\
\text { ture of } \\
\text { soapstock } \\
\text { proces- } \\
\text { sing, }{ }^{\circ} \mathrm{C}\end{array}$ & $\begin{array}{c}\text { Duraa } \\
\text { tion of } \\
\text { soapstock } \\
\text { process- } \\
\text { ing, min. }\end{array}$ & $\begin{array}{c}\text { Fatty } \\
\text { acid } \\
\text { yield, \% }\end{array}$ & $\begin{array}{c}\text { Neutrali- } \\
\text { zation number } \\
\text { of fatty acids, } \\
\text { mg KOH/g }\end{array}$ \\
\hline 1 & 55 & 40 & 48.1 & 99.5 \\
\hline 2 & 55 & 80 & 56.2 & 125.0 \\
\hline 3 & 55 & 120 & 84.3 & 128.0 \\
\hline 4 & 75 & 40 & 63.2 & 131.5 \\
\hline 5 & 75 & 80 & 65.1 & 145.7 \\
\hline 6 & 75 & 120 & 78.2 & 173.0 \\
\hline 7 & 95 & 40 & 79.2 & 180.0 \\
\hline 8 & 95 & 80 & 85.2 & 185.0 \\
\hline 9 & 95 & 120 & 90.1 & 189.2 \\
\hline
\end{tabular}

\begin{aligned}$\square & >90 \\ & <86 \\$\hline$\square & <76 \\$\hline$\square & <66 \\$\hline$\square & <56 \\ & <46\end{aligned}$

Duration of soapstock processing, min

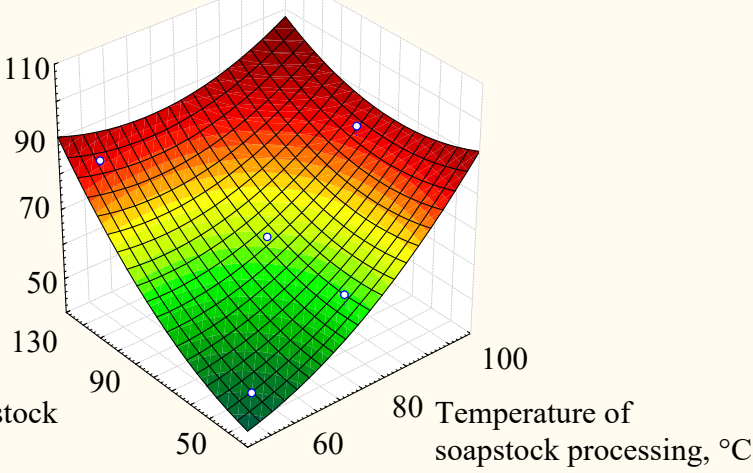

Fig. 2. Dependence of the fatty acid yield on the temperature and duration of soapstock processing with sulfuric acid (response surface)

By equation (5) and Fig. 3, 4, it is found that

Table 3 the temperature of soapstock processing also has

Estimated values of the yield and neutralization number of fatty acids a more significant effect on the neutralization number of fatty acids, i.e. their quality, than the process duration. Therefore, it is advisable to carry out the treatment process at the temperature of $(90-95){ }^{\circ} \mathrm{C}$. The duration has a negligible effect on the value of the neutralization number. Therefore, the following conditions can be considered ra-

Fig. 1, 2 show the response surface projection and the response surface, which is the dependence of the fatty acid yield on the temperature and duration of soapstock processing.

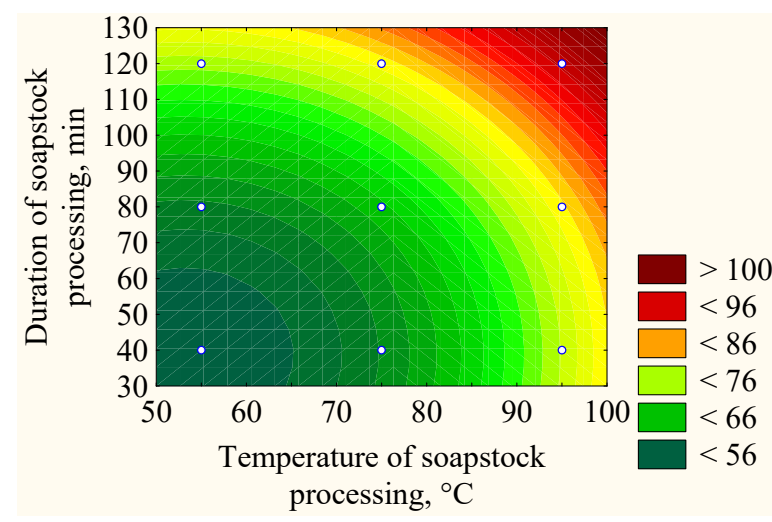

Fig. 1. Dependence of the fatty acid yield on the temperature and duration of soapstock processing with sulfuric acid (response surface projection)

By analyzing equation (3) and Fig. 1,2, it is found that the soapstock processing temperature has a more significant effect on the fatty acid yield than the duration. Thus, it is advisable to treat the soapstock at the temperature of $(90-95){ }^{\circ} \mathrm{C}$. The processing time of 40 minutes can be used, as under selected temperature conditions the duration of up to 90 minutes has practically no effect on the fatty acid yield. Further insignificant increase in the response function occurs only with a simultaneous increase in the duration and temperature of processing. Under the duration of 40 minutes and the temperature of $90{ }^{\circ} \mathrm{C}$, the fatty acid yield was $79.0 \%$.

Fig. 3, 4 show the response surface projection and the response surface, which is the dependence of the neutralization number of fatty acids on the temperature and duration of soapstock processing with sulfuric acid. tional: process temperature $(90-95){ }^{\circ} \mathrm{C}$, duration $40 \mathrm{~min}$. Under the duration of 40 minutes and the temperature of $90{ }^{\circ} \mathrm{C}$, the neutralization number of fatty acids was $180 \mathrm{mg} \mathrm{KOH} / \mathrm{g}$.

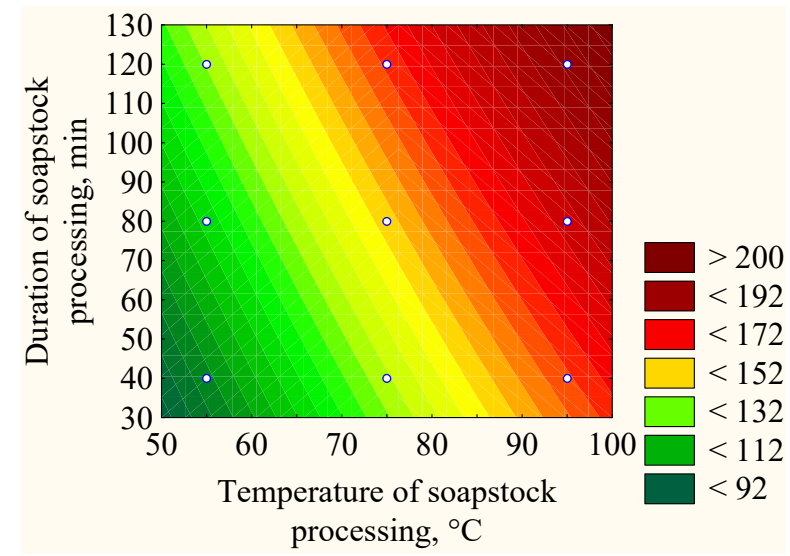

Fig. 3. Dependence of the neutralization number of fatty acids on the temperature and duration of soapstock processing with sulfuric acid (response surface projection)

Neutralization number, $\mathrm{mg} \mathrm{KOH} / \mathrm{g}$
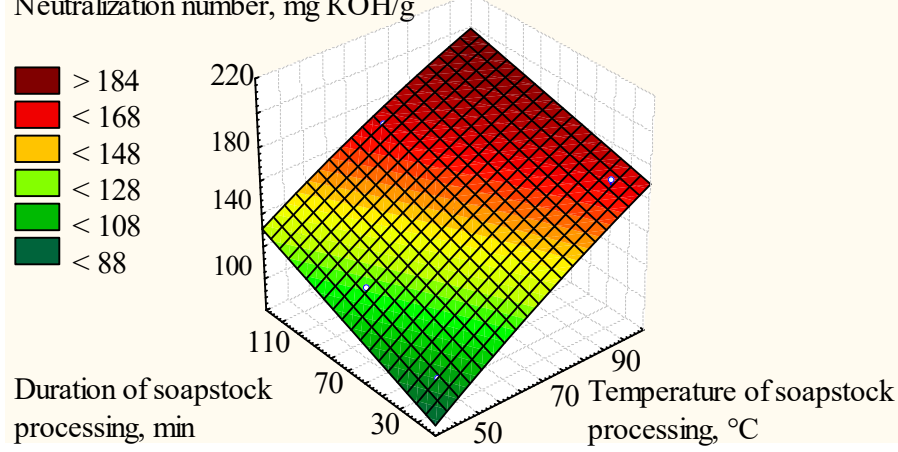
processing, min

Fig. 4. Dependence of neutralization number of fatty acids on the temperature and duration of soapstock processing with sulfuric acid (response surface) 
5. 3. Research of quality indicators and composition of fatty acids

Qualitative indicators of fatty acids obtained under the established rational conditions are determined. The corresponding data are given in Table 4 .

Table 4

Qualitative indicators of fatty acids obtained under rational conditions

\begin{tabular}{|c|c|}
\hline Indicator & Characteristics \\
\hline Color at the temperature of $20^{\circ} \mathrm{C}$ & Brown \\
\hline Odor & Specific to fatty acids \\
\hline $\begin{array}{c}\text { Mass fraction of moisture and volatile } \\
\text { substances, } \%\end{array}$ & 1.8 \\
\hline Mass fraction of total fat, $\%$ & 97.0 \\
\hline Cleavage depth, $\%$ oleic acid & 64.5 \\
\hline Presence of mineral acids & None \\
\hline
\end{tabular}

The obtained fatty acids are of high quality and correspond to the characteristics of fatty acids of light oils and modified fats, obtained without saponification, first grade according to DSTU 4860 (CAS 61788-66-7).

For comparative analysis, the composition of the obtained fatty acids and the experimental sample of sunflower oil was determined. The corresponding data are shown in Table 5.

Table 5

Composition of the obtained fatty acids and fatty acid composition of sunflower oil

\begin{tabular}{|c|c|c|c|}
\hline \multirow{2}{*}{ No. } & \multirow{2}{*}{ Fatty acid } & \multicolumn{2}{|c|}{ Mass fraction of fatty acid, $\%$} \\
\hline & & Obtained fatty acids & Sunflower oil \\
\hline 1 & Palmitic $\mathrm{C}_{16: 0}$ & 0 & 1.7 \\
\hline 2 & Palmitoleic $\mathrm{C}_{16: 1}$ & 0.7 & 0.2 \\
\hline 3 & Stearic $\mathrm{C}_{18: 0}$ & 6.9 & 3.6 \\
\hline 4 & Oleic $\mathrm{C}_{18: 1}$ & 37.4 & 30.9 \\
\hline 5 & Linoleic $\mathrm{C}_{18: 2}$ & 51.2 & 62.3 \\
\hline 6 & Linolenic $\mathrm{C}_{18: 3}$ & 0 & 0.1 \\
\hline 7 & Arachinic $\mathrm{C}_{20: 0}$ & 0.4 & 0.2 \\
\hline 8 & Gadoleic $\mathrm{C}_{20: 1}$ & 0.8 & 0.1 \\
\hline 9 & Behenic $\mathrm{C}_{22: 0}$ & 1.2 & 0.7 \\
\hline 10 & Lignoceric $\mathrm{C}_{24: 0}$ & 0.4 & 0.2 \\
\hline 11 & Myristic $\mathrm{C}_{14: 0}$ & 1.0 & 0 \\
\hline & Total & 100.000 & 100.0 \\
\hline
\end{tabular}

The composition of fatty acids obtained from soapstock after alkaline neutralization of sunflower oil has differences in comparison with the fatty acid composition of the sunflower oil sample. There is a correlation for fatty acids, the mass fractions of which are the largest (stearic, oleic, linoleic, behenic).

\section{Discussion of the results of studying the dependence of the influence of soapstock processing conditions on the efficiency of fatty acid production}

Rational conditions for processing soapstock obtained after alkaline neutralization of sunflower oil with sulfuric acid have been determined: process temperature (9095) ${ }^{\circ} \mathrm{C}$, duration $40 \mathrm{~min}$. The experimentally determined values of the response functions under rational conditions were: fatty acid yield - $79.0 \%$, neutralization number $180.0 \mathrm{mg} \mathrm{KOH} / \mathrm{g}$. Using the real values of the variation factors (temperature and duration of the process) according to equations (3) and (5), it is possible to predict both the yield and neutralization number, which characterizes the fatty acid quality with an error of not more than $6 \%$. Qualitative indicators of the obtained fatty acids correspond to the characteristics of fatty acids of light oils and modified fats, obtained without saponification, of the first grade according to DSTU 4860 (CAS 61788-66-7). The results of the study indicate the high quality of the isolated fatty acids.

This work differs from the existing [7, 9, 14-16] scientific researches on the extraction of fatty acids from soapstocks by the method of acid action by considering the most important technological parameters. The influence of soapstock processing conditions not only on the yield, but also on the quality of fatty acids, characterized by the neutralization number, was studied.

The neutralization number characterizes the purity of the product and is a constant for individual fatty acids. This value is used in technological calculations, when determining the amount of reagents, materials for obtaining products based on fatty acids. The neutralization number controls the conversion degree of fatty acids in further processing. The developed mathematical models make it possible to predict the neutralization number of fatty acids, which is necessary when calculating the required amount of fatty acids for further processing. These data are relevant for oil and fat enterprises and industries, which use fatty acids as the raw material.

With increasing temperature of soapstock treatment with sulfuric acid in the experimental range from 55 to $95{ }^{\circ} \mathrm{C}$ with the reaction time of 40 minutes, the fatty acid yield increases 1.6 times, with the duration of 80 minutes 1.5 times (Table 2). This indicator characterizes the intensity of soapstock decomposition. It is found that it is advisable to treat the soapstock at the temperature of $(90-95){ }^{\circ} \mathrm{C}$, because the maximum values of the response functions are achieved (fatty acid yield - up to $93.9 \%$, neutralization number - up to $192.9 \mathrm{mg} \mathrm{KOH} / \mathrm{g}$ ), which is confirmed by the data in Tables 2, 3 and Fig. 1-4.

The processing duration affects the efficiency of fatty acid extraction as follows. With increasing duration of processing in the experimental range from 40 to $120 \mathrm{~min}$ utes, only at the temperature of $55^{\circ} \mathrm{C}$, there is a significant increase in yield (1.75 times). But at this temperature, the yield values are the lowest in the experiment (up to $78.9 \%$ ). Under conditions of temperature increase to $95^{\circ} \mathrm{C}$ (within the limits of the experiment), prolongation of the process duration practically does not affect the yield (the growth of this indicator no more than 1.2 times). The corresponding data are shown in Table 2. Therefore, the rational duration of the process is 40 minutes (minimum within the experiment).

The composition of the obtained fatty acids is compared with the fatty acid composition of the sample of sunflower oil (Table 5). There is a correlation for fatty acids, the mass fractions of which are the largest (stearic, oleic, linoleic, behenic). Thus, fatty acids derived from soapstock contain $37.4 \%$ oleic acid, $51.2 \%$ linoleic acid; fatty acids from sunflower oil contain $30.9 \%$ oleic acid, $62.3 \%$ linoleic acid.

During the implementation of the research results in production, it is necessary to use the developed rational conditions (temperature of soapstock treatment with sulfuric acid $(90-95)^{\circ} \mathrm{C}$, duration $40 \mathrm{~min}$.). Changing the tempera- 
ture from 95 to $55^{\circ} \mathrm{C}$ reduces the efficiency of the process, which is expressed in a decrease in yield by 1.6 times, neutralization number - by 1.5 times, as shown in Table 2 .

The resulting fatty acids must be washed from sulfuric acid residues and checked for the presence of sulfate ions using $10 \%$ calcium chloride solution. Residual sulfuric acid content can distort the results of determining the neutralization number, composition and other quality indicators of fatty acids. Also, the presence of sulfuric acid can lead to the formation of unwanted reaction products and poor-quality products during the subsequent use of fatty acids.

The disadvantage of the study can be considered the use of sulfuric acid, which is dangerous from the safety point of view. But now this method of obtaining fatty acids from soapstocks is rational [7]. And, therefore, scientific developments in this field are relevant, as they contribute to solving the problem of utilization of soapstocks produced by oil and fat enterprises. Research aimed at increasing the efficiency and profitability of processing soapstocks into fatty acids is important. Because there is a wide range of applications of these compounds, including biodiesel production.

Promising areas of research are to determine the influence of the conditions of soapstock treatment with sulfuric acid on other indicators of fatty acids: saponification number, ether number. These data will be useful for companies that use fatty acids in the production of soap, esters, etc. Also of interest is the further use of fatty acids obtained under different conditions in the processes of formation of biodiesel fuel and the study of its indicators. This will allow determining rational technological parameters in the fatty acid production from soapstocks in order to obtain biodiesel.

\section{Conclusions}

1. On the basis of experimental researches, the quality indicators of the experimental sample of soapstock, obtained as a result of alkaline neutralization of sunflower oil, are determined. Soapstock has the following indicators: mass fraction of moisture $-15.4 \%$, mass fraction of total fat $71.9 \%$, mass fraction of fatty acids $-64.5 \%$, mass fraction of neutral fat $-7.4 \%$. The experimental sample of soapstock complies with DSTU 5033 (CAS 68952-95-4).

2. The dependence of the yield and neutralization number of fatty acids on the conditions of soapstock processing with sulfuric acid is identified. Relevant mathematical models are obtained. Rational parameters of soapstock processing are determined: process temperature $(90-95){ }^{\circ} \mathrm{C}$, duration $40 \mathrm{~min}$. The fatty acid yield under these conditions was $79.0 \%$, the neutralization number was $180.0 \mathrm{mg} \mathrm{KOH} / \mathrm{g}$.

3 . The quality indicators and composition of fatty acids obtained under the established rational conditions are investigated. Fatty acids have the following indicators: mass fraction of moisture and volatile substances $-1.8 \%$, mass fraction of total fat $-97.0 \%$, cleavage depth $-64.5 \%$ oleic acid, the presence of mineral acids - no. Fatty acids correspond to fatty acids of the first grade, obtained without saponification, in accordance with DSTU 4860 (CAS 61788-66-7). The comparative analysis of the composition of fatty acids from soapstock and fatty acid composition of sunflower oil was performed. There is a correlation for fatty acids with the largest mass fractions. Thus, fatty acids derived from soapstock contain $37.4 \%$ oleic acid and $51.2 \%$ linoleic acid; fatty acids from sunflower oil contain $30.9 \%$ oleic acid and $62.3 \%$ linoleic acid. The obtained mathematical models allow carrying out the process of soapstock processing to obtain fatty acids under rational conditions. Data on the neutralization number make it possible to predict the quality of fatty acids obtained at various technological parameters, as well as to use these data in further processes of fatty acid application. Recycling large-tonnage waste - soapstock - will help reduce the negative impact of the oil and fat industry on the environment.

\section{References}

1. Sytnik, N., Kunitsia, E., Mazaeva, V., Chernukha, A., Kovalov, P., Grigorenko, N. et. al. (2020). Rational parameters of waxes obtaining from oil winterization waste. Eastern-European Journal of Enterprise Technologies, 6 (10 (108)), 29-35. doi: https:// doi.org/10.15587/1729-4061.2020.219602

2. Strizhenok, A., Ivanov, A. (2021). Monitoring of Air Pollution in the Area Affected by the Storage of Primary Oil Refining Waste. Journal of Ecological Engineering, 22 (1), 60-67. doi: https://doi.org/10.12911/22998993/128873

3. Sytnik, N., Kunitsa, E., Mazaeva, V., Chernukha, A., Bezuglov, O., Bogatov, O. et. al. (2020). Determination of the influence of natural antioxidant concentrations on the shelf life of sunflower oil. Eastern-European Journal of Enterprise Technologies, 4 (11 (106)), 55-62. doi: https://doi.org/10.15587/1729-4061.2020.209000

4. Kovari, K., Denise, J., Hollo, J. (2006). Seed crushing, oil refining and environmental problem. Olaj. Szap., 45 (2), 45-52.

5. Teslenko, A., Chernukha, A., Bezuglov, O., Bogatov, O., Kunitsa, E., Kalyna, V. et. al. (2019). Construction of an algorithm for building regions of questionable decisions for devices containing gases in a linear multidimensional space of hazardous factors. Eastern-European Journal of Enterprise Technologies, 5 (10 (101)), 42-49. doi: https://doi.org/10.15587/1729-4061.2019.181668

6. Khedkar, R., Singh, K. (2018). Food Industry Waste: A Panacea or Pollution Hazard? Paradigms in Pollution Prevention, 35-47. doi: https://doi.org/10.1007/978-3-319-58415-7_3

7. Molchenko, S. M., Demydov, I. M., Vied, V. Ye. (2015). Oderzhannia zhyrnykh kyslot z soapstoku shliakhom rozkladannia myla karbonatnoiu kyslotoiu. Visnyk Natsionalnoho tekhnichnoho universytetu «KhPI», 7 (1116), 76-82. Available at: http:// repository.kpi.kharkov.ua/bitstream/KhPI-Press/15991/1/vestnik_HPI_2015_7_Molchenko_Oderzhannia.pdf

8. Levterov, A. M., Levterov, A. A. (2018). Thermodynamic properties of fatty acid esters in some biodiesel fuels. Functional Materials, 25 (2), 308-312. doi: https://doi.org/10.15407/fm25.02.308

9. Wang, Y., Ke, L., Peng, Y., Yang, Q., Du, Z., Dai, L. et. al. (2020). Characteristics of the catalytic fast pyrolysis of vegetable oil soapstock for hydrocarbon-rich fuel. Energy Conversion and Management, 213, 112860. doi: https://doi.org/10.1016/ j.enconman.2020.112860 
10. Kondratenko, O. M., Vambol, S. O., Strokov, O. P., Avramenko, A. M. (2015). Mathematical model of the efficiency of diesel particulate matter filter. Naukovyi Visnyk Natsionalnoho hirnychoho universytetu, 6, 55-61. Available at: https://www.scopus. com/record/display.uri?eid=2-s2.0-84959270723\&origin=inward\&txGid=a41878294c3ff8332ad19932a6bd41ff

11. Tripathi, S., Subramanian, K. A. (2017). Experimental investigation of utilization of Soya soap stock based acid oil biodiesel in an automotive compression ignition engine. Applied Energy, 198, 332-346. doi: https://doi.org/10.1016/j.apenergy.2017.02.057

12. Chervakov, V. O., Filinskaja, T. G., Kapiton, V. O. (2009). The methods of transesterification feedstock containing of fat by alcoholysis. Problems of Chemstry and Chemical Technologies, 4, 72-79.

13. Sánchez Faba, E. M., Ferrero, G. O., Dias, J. M., Eimer, G. A. (2019). Alternative Raw Materials to Produce Biodiesel through Alkaline Heterogeneous Catalysis. Catalysts, 9 (8), 690. doi: https://doi.org/10.3390/catal9080690

14. Ostafin, M. M., Lenik, E., Bulski, K., Kielbasa, P., Drozdz, T., Trzyniec, K., Tarniowy, A. (2018). Recovering of the long-chain fatty acids from soapstock. Przemysl chemiczny, 97 (3), 387-392. doi: https://doi.org/10.15199/62.2018.3.9

15. Abdikamalova, A. B., Sharipova, A. Sh., Artikova, G. N., Seytnazarova, O. M., Ismaylov, B. M. (2016). Sposoby vydeleniya zhirnyh kislot iz soapstokov. Sovremennye innovatsii, 6 (8), 12-14. Available at: https://cyberleninka.ru/article/n/sposoby-vydeleniyazhirnyh-kislot-iz-soapstokov

16. Demidov, I., Sytnik, N., Mazaeva, V. (2014). Sunflower and problem alternative fuel in Ukraine. Naukovo-tekhnichnyi biuleten Instytutu oliynykh kultur NAAN, 21, 137-146.

17. Pospelov, B., Meleshchenko, R., Asotskyi, V., Petukhova, O., Gornostal, S., Harbuz, S. (2019). Development of a self-adjusting method for calculating recurrent diagrams in a space with a scalar product. EUREKA: Physics and Engineering, 5, 10-18. doi: https://doi.org/10.21303/2461-4262.2019.00981

18. Chernukha, A., Teslenko, A., Kovalov, P., Bezuglov, O. (2020). Mathematical Modeling of Fire-Proof Efficiency of Coatings Based on Silicate Composition. Materials Science Forum, 1006, 70-75. doi: https://doi.org/10.4028/www.scientific.net/msf.1006.70 